\title{
EEG Subband Analysis using Approximate Entropy for the Detection of Epilepsy
}

\author{
G.R.Kiranmayi*, V. Udayashankaras \\ *JSS Research Foundation, Mysore, Karnataka,India, kiran_gr@hotmail.com \\ ${ }^{\$}$ Department of IT, SJCE, Mysore, Karnataka, India, v_udayashankara@yahoo.co.in
}

\begin{abstract}
Epilepsy is a neurological disorder which affects the nervous system. Epileptic seizures are due to sudden hyperactivity in certain parts of the brain. Electroencephalogram (EEG) is the commonly used modality for the detection of epilepsy. Automatic seizure detection helps in diagnosis and monitoring of epilepsy especially during long term recordings of EEG. This paper presents non linear analysis of EEG for the detection of epilepsy using approximate entropy (ApEn). The proposed method involves ApEn measured from EEG subbands applied as features to an artificial neural network (ANN) classifier. The ApEn measured from delta, theta, alpha, beta and gamma subbands of normal EEG, ictal and inter ictal EEGs are used as features. In the present work detection of epilepsy is considered as a two class problem. In the first case the classification is done between normal and ictal EEGs and in the second case, classification is done between normal and inter ictal EEGs. For both cases artificial neural networks with back propagation training are used as classifiers. The classification accuracy of 100\% is obtained for normal and ictal groups and that of $98.9 \%$ is obtained for normal and inters ictal EEGs.
\end{abstract}

Keywords: Electroencephalogram (EEG), ictal and inter ictal EEG, approximate entropy, neural network classifier.

\section{Introduction}

Electroencephalogram (EEG) is clinically significant in treating patients with various neurological disorders. EEG is a record of electric signals generated by the synchronous action of brain cells. Normally EEG is recorded from the scalp. EEG signal is non-stationary. Epilepsy is one of the commonly observed neural disorders among adults and children. EEG is the most useful and cost effective tool for the study of epilepsy. Epileptic seizures are due to sudden excessive electrical discharges in a group of neurons. Epilepsy affects more than $1 \%$ of the world's population. The occurrence of epileptic seizure is random and affects the normal functioning of the brain. The EEG recordings of patients suffering from epilepsy are grouped into inter ictal and ictal. Signal recorded between epileptic seizures is called inter ictal EEG and the signal recorded during an epileptic seizure is called ictal EEG [1], [2].

Diagnosis of epilepsy generally involves visual inspection of the EEG recordings by a neurologist. This is difficult for long term recordings. Subtle changes in EEG pattern is difficult to detect visually even by an experienced neurologist. Automatic seizure identification has challenged researchers for more than two decades. Various techniques have been proposed for the detection and prediction of epileptic seizure in EEG. Wavelet transform based techniques are found useful in the detection of epileptic seizures [3], [4], [5]. AR modelling is preferred for real-time processing and analysis of epileptic EEG [6], [7]. M. Han and L. Sun proposed an EEG signal classification method based on relevance vector machine (RVM) and AR model [8]. S.Guzman et al. explored the advantages of Independent-Component Analysis (ICA) and State-Space Modelling (SSM) in the analysis of EEG [9]. M. T. Acar analyzed neuronal dynamics before and during epileptic seizures using adaptive multivariate autoregressive model [10]. Empirical mode decomposition (EMD) based method is proposed by L. Orosco [11].

Several processing techniques proposed and available in the literature assume that the EEG is generated by a highly complex linear system, but functioning of brain is non-linear. As non-linearity in the brain is introduced even at the cellular level, non-linear dynamical analysis technique may be a better approach than a traditional linear method to understand the EEG dynamics [12].

ApEn indicates complexity of a signal. This parameter helps in understanding the non linearity of the signal and hence the non linear dynamics of the underlying generating system. ApEn is a very good measure for understanding biological signals [13], [14]. EEG becomes more rhythmic during epileptic seizure. This property is used in detecting seizure in the proposed method. ApEn value indicates the irregularity of a signal; higher value indicates more complexity or irregularity. ApEn value drops during seizure. This property is used by some researchers [15], [16], [17]. ApEn measured in wavelet domain can also discriminate epileptic EEG from normal EEG [18], [19], [20]. ApEn is used to find localization of epileptic foci [21]. 


\section{Approximate Entropy}

The ApEn, first proposed by Pincus [13], is a statistic used to quantify the unpredictability of fluctuations in both deterministic and stochastic signals. It is a measure which can quantify the complexity or irregularity of the signal. ApEn is less sensitive to noise and can be used for short-length data. In addition, it is resistant to short strong transient interferences such as spikes. A lower value of ApEn indicates that the time series is more rhythmic; a higher value indicates more randomness.

Two parameters are to be fixed to calculate ApEn, $r$ and $\mathrm{m}$. The parameter $r$ is the similar tolerance or filter factor. The general procedure is to set $r$ to be 0.2 times the standard deviation of the data. But for comparison of two data sets $r$ value should be same for both data sets [14]. The parameter $m$ is the "embedding dimension". Generally the value of $m$ is chosen to be 1,2 , or 3 . The input data for the computation of ApEn is a time-series with typically between 100 and 5000 samples [5].

The algorithm for calculating the ApEn of a time series $\mathrm{X}$ is as follows.

1 consider the sequence, $X=x(1), x(2), x(3) \ldots \ldots x(N)$ with $\mathrm{N}$ data points,

2 let $x(i)$ be a subsequence of $X$ such that $x(i)=[x(i), x(i+1), \ldots, x(i+m-1)]$

for $1 \leq i \leq(N-m)$, where $m$ represents the samples in the sub sequence.

3 define the distance $d\left(x_{i}, x_{j}\right)$ between two vectors $x_{i}$ and $x_{j}$ such that

$$
d\left(x_{i}, x_{j}\right)=\max _{k=1,2, \sim m}|X(i+k-1)-X(j+k-1)|
$$

4 for a given vector count the number $x_{j}, j=1,2, \ldots . . N-m+1, j \neq i$ such that $d\left(x_{i}, x_{j}\right) \leq r$ let this count be $N^{m}(i)$, where $r$ the predefined threshold.

compute the ratio $C_{r}^{m}(i)=N^{m}(i) / N-m+1$

5 compute the natural $\operatorname{logarithm}$ of $C_{r}^{m}(i)$ and find the average over all values of $i$, $\emptyset^{m}(r)=\frac{1}{N-m+1} \sum_{i=1}^{N-m+1} \ln C_{r}^{m}(i)$

6 repeat above steps to calculate $C_{r}^{m+1}(i)$ and $\emptyset^{m+1}(r)$ by increasing the dimension to $m+1$

7 ApEn is calculated as $A p E n=\emptyset^{m}(r)-\emptyset^{m+1}(r)$.

\subsection{Dataset description:}

\section{Data And Methodology}

The standard data set described by Andrzejak et al [22] is used in the present work. This dataset consists of five subsets Z, O, N, F, and S. Each subset consists of 100 single-channel EEG segments of $23.6 \mathrm{~s}$ duration. The signals are sampled at 173.61 samples per second with 16-bit resolution. Sets $Z$ and $O$ consists of EEG recordings acquired from the scalp of five healthy volunteers, with eyes open and closed, respectively. Z and $\mathrm{O}$ have been recorded extra cranially. Subsets $\mathrm{N}$ and $\mathrm{F}$ consists of Inter-ictal EEGs (seizure free interval). S contains ictal EEG (EEG recorded during seizure), sets N, F, and S have been recorded intra cranially. The subsets Z, S, F are used for the present work. Time domain plots of subbands of normal EEG, inter ictal EEG and inter ictal EEG are shown in Fig. 1a, Fig.1b and Fig.1c respectively.

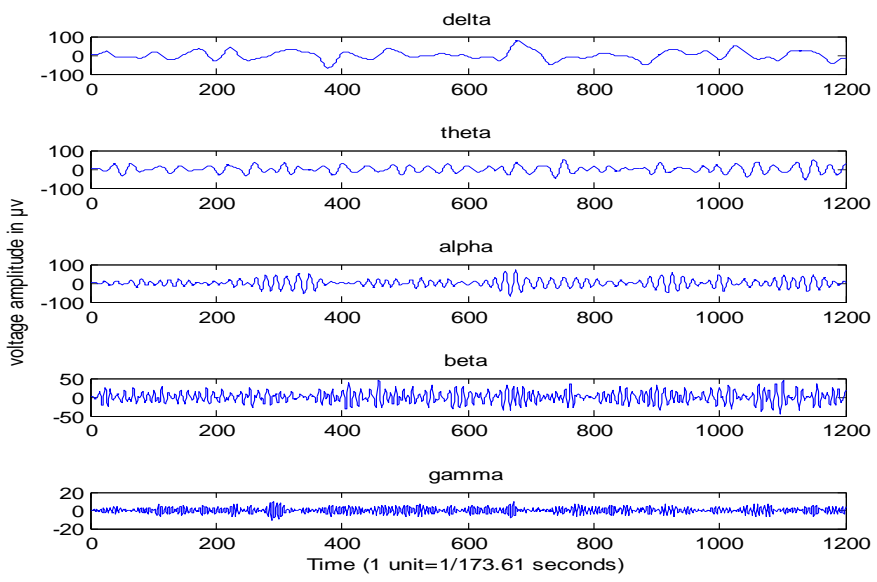

Fig.1a Subbands of a sample normal EEG in time domain 

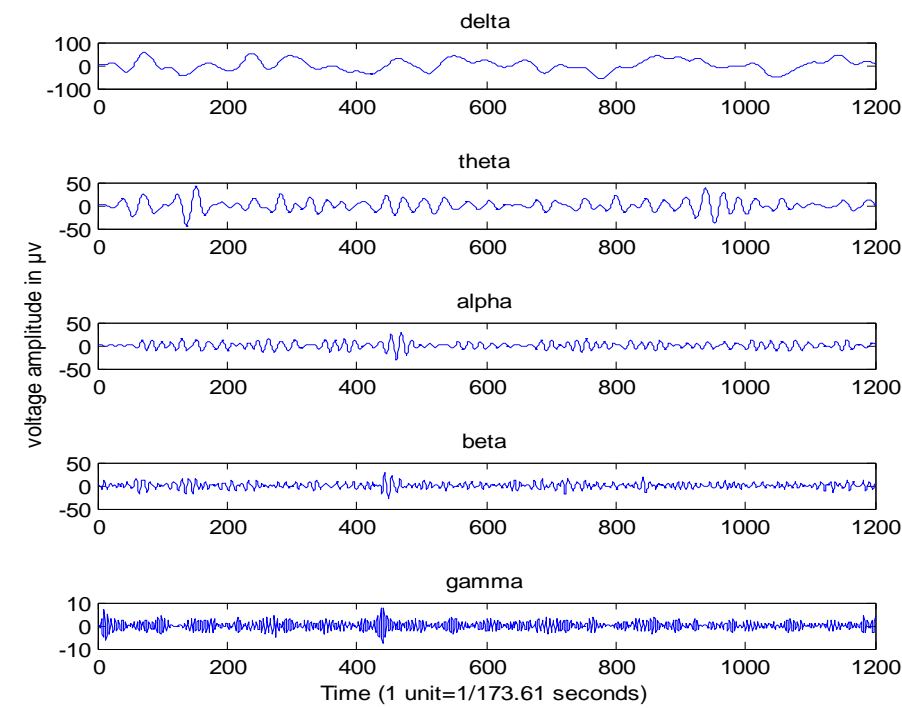

Fig.1b Subbands of a sample inter ictal EEG in time domain

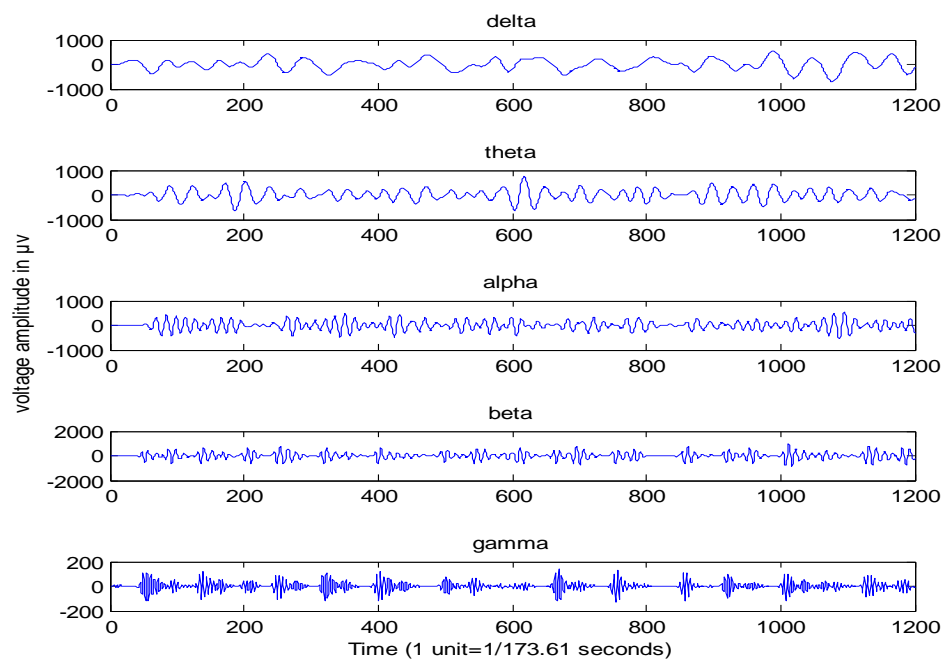

Fig.1c Subbands of a sample ictal EEG in time domain

\subsection{Classifier}

Artificial neural networks are computing systems made up of large number of simple interconnected processing elements called neurons. ANNs are widely used tool for classification. The advantages of neural networks are that, they are self adaptive in learning, robustness, self organization, and generalization capability. They are data driven and can approximate any function with an arbitrary accuracy [16]. In the present work, a three layer feed-forward back propagation neural network is trained with ApEn feature vectors to detect and classify normal, inter ictal and ictal EEGs. ApEn of delta, theta, alpha, beta and gamma subbands are computed separately and combined as a feature vector for each class. The schematic of the artificial neural network is shown in Fig. 2. 


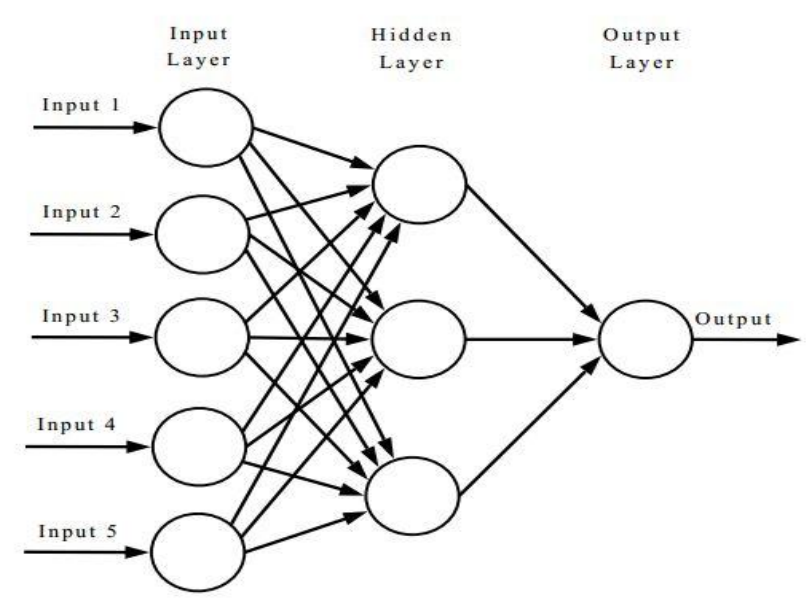

Fig.2 Schematic of a feed forward artificial neural network.

The neural network is trained using back propagation algorithm. In this method, the weights are randomly chosen and the ANN output is compared to the desired output. If the error is more, the connecting weights are modified. This process is continued until the error is less than a preset threshold. Performance of an ANN is evaluated by Sensitivity (SE), Specificity (SP) and Classification accuracy (CA). These parameters are defined as follows

Sensitivity, $S E(\%)=\frac{T_{C P}}{T_{A P}} \times 100$

$T_{C P}=$ total number of correctly detected positive patterns

$T_{A P}=$ total number of actual positive patterns

Specificity, $S P(\%)=\frac{T_{C N}}{T_{A N}} \times 100$

$T_{C N}=$ total number of correctly detected negative patterns

$T_{A N}=$ total number of actual negative patterns

Classification Accuracy, $C A(\%)=\frac{T_{C D P}}{T_{A P P}} \times 100$

$T_{C D P}=$ total number of correctly detected patterns

$T_{A P P}=$ total number of applied patterns

\section{Results}

ApEn values are computed for the datasets Z, S and F. From each data set, 300 EEG segments of 1024 samples are used for the computation. The values of $\mathrm{m}$ and $\mathrm{r}$ used for the computation of ApEn are 2 and 0.1 respectively. Butterworth filter is used to separate the EEG subbands. ApEn of delta, theta, alpha, beta and gamma subbands are computed separately and combined as a feature vector for each class. ApEn values of first 100 EEG segments used for the analysis in each class are plotted in Figures 3 and 4 respectively. A set of 300 feature vectors corresponding to classes $\mathrm{Z}$ and $\mathrm{S}$ are used for training and testing the BPNN. Out of 300, 70\% is used for training and $30 \%$ is used for validation and testing. A Classification accuracy of $100 \%$ is obtained. A Similar procedure is used for the classes Z and F. A classification accuracy of $98.9 \%$ is obtained. The error curves for the Z-S classifier and Z-F classifier are shown in Figures 5 and 6 respectively. 

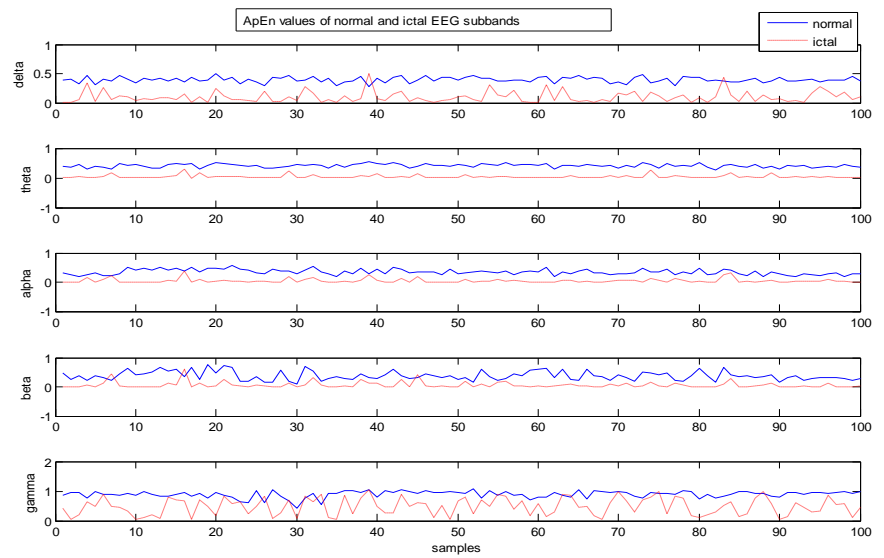

Fig. 3 Sample ApEn values of the normal and ictal EEG sub bands
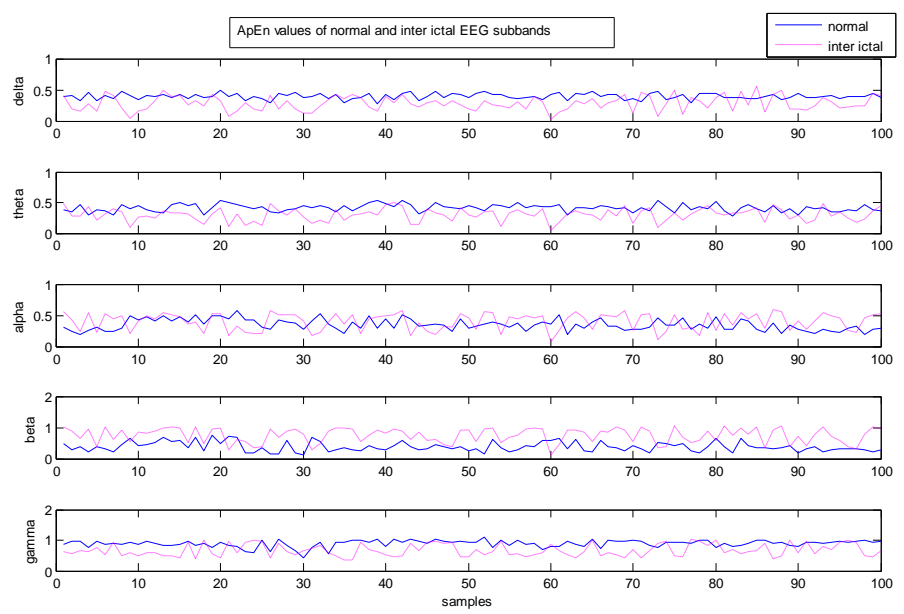

Fig.4 Sample ApEn values of the normal and inter ictal EEG sub bands

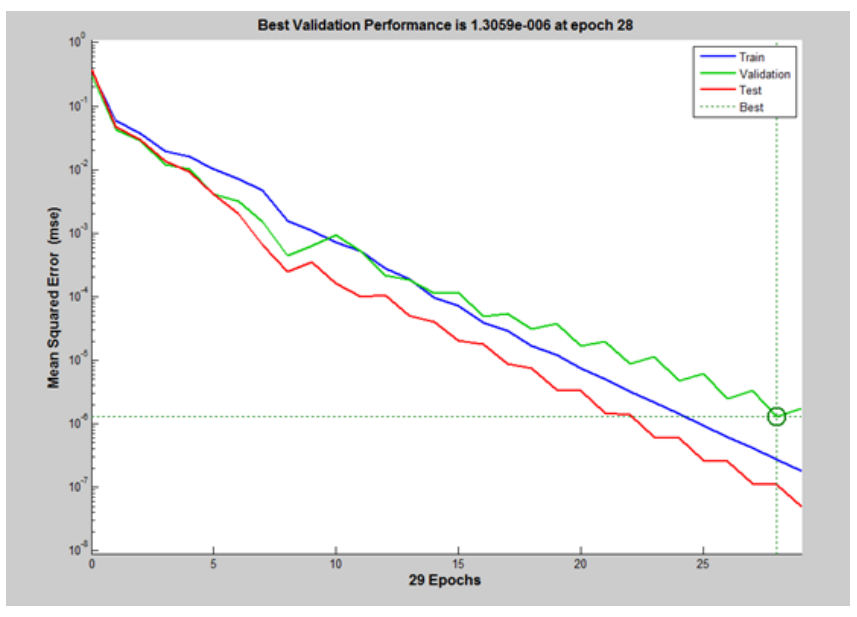

Fig.5 Mean square error curve for the Z, S classification 


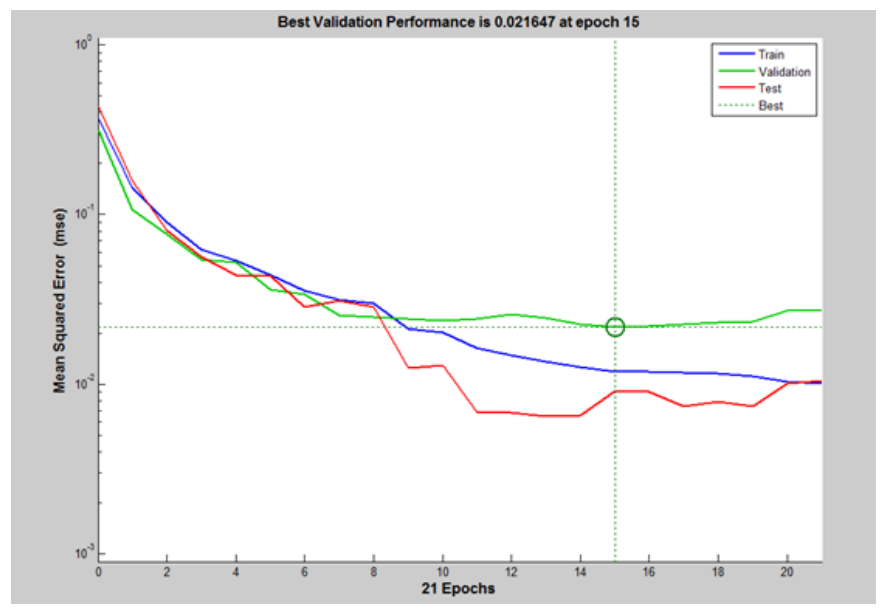

Fig.6 Mean square error curve for the Z, F classification

TABLE 1 Performance of the proposed classifier

\begin{tabular}{|c|c|c|c|}
\hline Class & Classification & Sensitivity & Specificity \\
\hline Normal- ictal $(\mathrm{Z}, \mathrm{S})$ & $100 \%$ & $100 \%$ & $100 \%$ \\
\hline Normal - inter ictal & $98.9 \%$ & $97.4 \%$ & $100 \%$ \\
\hline
\end{tabular}

\section{Discussion}

ApEn which is a non linear statistic helps in understanding the non linear dynamics of the signal there by helps to understand the dynamics of the underlying system which generates the signal. Our approach is to compute the ApEn of the EEG sub bands and to use as features. The ApEn computed from delta, theta, alpha, beta and gamma sub bands are combined and used as feature vector.

Table 2 presents a comparison of our method and the methods proposed by other researchers. For comparison, the methods that are evaluated using the same data set are included.

TABLE 2 Comparison of the performance of the methods proposed by other

\begin{tabular}{ccc}
\hline \hline & Methods proposed by other Researchers & \\
\hline Authors & Dataset & Classification Accuracy \% \\
\hline Nigam et al [23] & $\mathrm{Z}, \mathrm{S}$ & 97.2 \\
Srinivasan et al [24] & $\mathrm{Z}, \mathrm{S}$ & 99.6 \\
Kannathal et al.[25] & $\mathrm{Z}, \mathrm{S}$ & 92.22 \\
Polat et al [26] & $\mathrm{Z}, \mathrm{S}$ & 98.72 \\
Subasi [27] & $\mathrm{Z}, \mathrm{S}$ & 95 \\
\hline Alexandros T. Tzallas et al[28] & Z, S & 100 \\
Srinivasan et al [16] & Z, S & $99.35-100$ \\
\hline Dataset & Proposed method & Classification Accuracy \% \\
\hline Z,S & & 100 \\
Z,F & & 98.9 \\
\hline
\end{tabular}

The proposed methodology achieves $100 \%$ classification accuracy in detecting ictal EEG from normal EEG. Methods proposed in [16] and [28] also claim 100\% accuracy. Srinivasan et al[16] used the approximate entropy as the parameter and ELMAN Neural network and Probabilistic Neural network as classifiers. The approach of the proposed method is computationally simple and different. We have used a three layer ANN with back propogation, and ApEn computed from EEG subbands are used as features. The method proposed can also detect inter ictal EEGs from normal EEG. Accuracy of $98.9 \%$ is obtained in classifying normal and inter ictal EEGs. Related works [18], [19], [20] used ApEn as a feature, but exclusive Z-F classification accuracy is not available.

\section{Conclusion}

We have presented a new approach for detecting epileptic EEG using ApEn, a statistic which helps to quantify the non linear dynamics. During ictal period EEG pattern consists of rhythmical waveforms for a wide variety of frequencies. This change in EEG pattern is explored in the present work. We have utilized the property that the value of the ApEn drops sharply during an epileptic seizure. We have proved that ApEn values 
computed from EEG subbands delta, theta, alpha, beta, gamma can be used as feature set in detecting ictal and inter ictal EEGs.These features when combined can give better result as compared to the features extracted from EEG alone. An extension of the proposed method is to detect preictal EEG so that prediction of seizure is possible.

\section{References}

[1] Saeid Sanei and J.A Chambers, "EEG Signal Processing", John Wiley and Sons Ltd. 2007.

[2] G.R.Kiranmayi, V.Udayashankara, "Neural Network classifier for the detection of Epilepsy", proceedings of the International IEEE Conference on Circuits, controls and communications, CCUBE 2013, pp 1-4,27 - 28, December 2013.

[3] H. Adeli, S.G.Dastidar, and N. Dadmehr, "A Wavelet-Chaos Methodology for Analysis of EEGs and EEG Subbands to Detect Seizure and Epilepsy”, IEEE Transactions on Biomedical Engineering, Vol. 54, No. 2, pp 205-211, February 2007.

[4] S.G.Dastidar, H. Adeli and N. Dadmehr, "Mixed-Band Wavelet-Chaos-Neural Network Methodology for Epilepsy and Epileptic Seizure Detection", IEEE Transactions on Biomedical Engineering, Vol. 54, N0. 9, pp 1545-1551, September 2007.

[5] S.G.Dastidar, H. Adeli, and N. Dadmehr, "Principal Component Analysis-Enhanced Cosine Radial Basis Function Neural Network for Robust Epilepsy and Seizure Detection”, IEEE Transactions on Biomedical Engineering, Vol. 55, No. 2, pp 512-518, February 2008.

[6] Ozbeyaz, A., Gursoy M.I. Coban R ., "Regularization and Kernel Parameters Optimization based on PSO algorithm in EEG Signals Classification with SVM", Proceedings of the IEEE Conference on Signal Processing and Communications Applications (SIU), pp 399 - 402, 2011.

[7] Chisci, L. Mavino, A. Perferi, G. Sciandrone, M.Anile, C. Colicchio, G. Fuggetta, F., "Real-Time Epileptic Seizure Prediction Using AR Models and Support Vector Machines ”, IEEE Transactions on Biomedical Engineering Vol. 57 , No. 5 , pp 1124 -1132 2010 .

[8] M. Han, Leilei Sun,"EEG signal classification for epilepsy diagnosis based on AR model and RVM", Proceedings of the International Conference on Intelligent Control and Information Processing (ICICIP), pp 134 -139, 2010.

[9] S.Guzman, U. Heute, A. Galka, A.Stephani, U, “Application of State-Space Modeling to Instantaneous Independent- Component Analysis", Proceedings of the 4th International Conference on Biomedical Engineering and Informatics (BMEI), Vol. 2, pp 640 643, 2011.

[10] M. T. Acar, Z.A. Worrell, G. Makeig, S, "Modeling Cortical Source Dynamics and Interactions during Seizure", Proceedings of International Conference of the IEEE Engineering in Medicine and Biology Society, EMBC, pp 1411 - 1414, 2011.

[11] L. Orosco, A. G. Correa, and Eric Laciar, "Multiparametric Detection of Epileptic Seizures using Empirical Mode Decomposition of EEG Records", Proceedings of $32^{\text {nd }}$ Annual International Conference of the IEEE EMBS Buenos Aires, Argentina, pp 951-954, August 31 - September 4, 2010.

[12] Daniel A, Christopher J. James, and Roberto Hornero, "Non-linear Analysis of Intracranial Electroencephalogram Recordings with Approximate Entropy and Lempel-Ziv Complexity for Epileptic Seizure Detection" Proceedings of the 29th Annual International Conference of the IEEE EMBS, France, pp 1953 - 1956, August 2007.

[13] S.M.Pincus, "Approximate entropy as a measure of system complexity", Proceedings of National Academy of Science, USA, vol. 88, pp 2297-2301, 1991.

[14] S.M. Pincus, “Approximate Entropy: A complexity measure for Biological Time series data", Proceedings of the IEEE conference, pp 35-36, 1991.

[15] Radhakrishnan N, Gangadhar B.N, "Estimating regularity in epileptic seizure time-series data, A complexity-measure approach", IEEE Engineering in Medicine and Biology magazine, Vol. 17, pp 89-94, 1998.

[16] Vairavan Srinivasan, Chikkannan Eswaran, and N. Sriraam, “Approximate Entropy-Based Epileptic EEG Detection Using Artificial Neural Networks", IEEE Transactions on Information Technology in Bio Medicine, Vol.11, No. 3, pp 288-295, May 2007.

[17] Giorgos Giannakakis, Vangelis Sakkalis, Matthew Pediaditis, Christina Farmaki, Pelagia Vorgia, and Manolis Tsiknakis, "An approach to absence epileptic seizures detection using Approximate Entropy", Proceedings of International Conference of the IEEE EMBS Osaka, Japan, pp 413 - 416, July 2013.

[18] Chun-mei Wang, Jun-zhong Zou1, Jian Zhang, Zhi-suo Zhang, Chong-ming Zhang, “Classifying Detection of Epileptic EEG Based on Approximate Entropy in Wavelet Domain", Proceedings of the IEEE conference on Bio medical Engineering and Informatics, pp 1-5, 2009.

[19] Guo L, Rivero D, Pazos A., "Epileptic seizure detection using multiwavelet transform based approximate entropy and artificial neural networks", Journal of Neuroscience Methods, pp156-163, October 2010.

[20] Hamed Vavadi, Ahmad Ayatollahi, Ahmad Mirzaei, "A wavelet-approximate entropy method for epileptic activity detection from EEG and its sub-bands", Journal Biomedical Science and Engineering, pp 1182-1189, 2010.

[21] Zhen Zhang, Yi Zhou, Tian Mei, Yi Zhou, Shouhong Du, Xianghua Tian, Ziyi Chen, "Localization of Epileptic Foci Based on Scalp EEG and Approximate Entropy", Proceedings of the IEEE Conference on Biomedical Engineering and Informatics (BMEI), pp 240-244, 2013.

[22] R. G. Andrzejak, K. Lehnertz, F. Mormann, C. Rieke, P. David, andC. E. Elger, "Indications of nonlinear deterministic and finitedimensional structures in time series of brain electrical activity: Dependence on recording region and brain state," Physical Review E: Statistical, Nonlinear, and Soft Matter Physics, Vol. 64, pp. 061907-1-061907-8, 2001.

[23] V. P. Nigam and D. Graupe, “A neural-network-based detection of epilepsy”, Neurology Research, vol. 26, no. 6, pp. 55-60, 2004

[24] V. Srinivasan, C. Eswaran, and N. Sriraam, "Artificial neural network based epileptic detection using time-domain and frequency domain features,",Journal of Medical Systems, vol. 29, No. 6, pp. 647-660, 2005.

[25] N. Kannathal, M. L. Choo, U. R. Acharya, and P. K. Sadasivan, "Entropies for detection of epilepsy in EEG," Computer Methods and Programs in Biomedicine,Vol. 80, No. 3, pp 187-194, December 2005.

[26] K. Polat and S. G"unes, "Classification of epileptiform EEG using a hybrid system based on decision tree classifier and fast Fourier transform," Applied Mathematics and Computation., vol. 32, no. 2, pp. 625-631, 2007.

[27] A. Subasi, "Signal classification using wavelet feature extraction and a mixture of expert model ", Expert Systems with Applications , vol. 32, No. 4, pp 1084-1093, 2007.

[28] A.T.Tzallas, M G. Tsipouras, and D I. Fotiadis, "Epileptic Seizure Detection in EEGs Using Time-Frequency Analysis", IEEE Transactions on Information Technology in Biomedicine, Vol. 13 No.5, pp 703-710, September 2009. 